\title{
Epidemiologia das helmintoses pulmonares e gastrintestinais de bezerros em região de baixada do Estado do Rio de Janeiro ${ }^{1}$
}

\author{
Manoel Pimentel Neto ${ }^{2}$ e Adivaldo Henrique da Fonseca ${ }^{3}$
}

\begin{abstract}
Pimentel Neto M. \& Fonseca A.H. 2002. [Epidemiology of pulmonary and gastrintestinal helmintoses in calves in the lowland of the state of Rio de Janeiro.] Epidemiologia das helmintoses pulmonares e gastrintestinais de bezerros em região de baixada do Estado do Rio de Janeiro. Pesquisa Veterinária Brasileira 22(4):148-152. Depto Epidemiologia e Saúde Pública, Universidade Federal Rural do Rio de Janeiro, Seropédica, RJ 23851-970, Brazil. E-mail: adivaldo@ufrrj.br

The epidemiology of pulmonary and gastrintestinal helmintoses of calves has been studied in cross-bred Zebu-Friesian calves, 6 to 9 month old, with natural infections. The calves were maintained in permanent pasturing in the lowland, with climate Aw, in the state of Rio de Janeiro. The experiments had the duration of 24 months. At the end the animals were bearer of natural infection of several helminth species. The research was based on fecal analysis and necropsies of at least four calves every 28 days. The animals maintained in permanent pasturing showed tendency to have larger helminth populations in autumn and spring. The graphical representation of an ellipse, obtained through the relationship of total rainfall and average minimum temperature per month, was effective in demonstrating the potential for clinical parasitism in autumn and spring.
\end{abstract}

INDEX TERMS: Epidemiology, pulmonary and gastrintestinal helmintoses, Haemonchus placei, Cooperia sp, Oesophagostomum radiatum, calves.

RESUMO.- Foi estudada a epidemiologia das helmintoses pulmonares e gastrintestinais em bezerros mestiços (Zebu $\mathrm{x}$ Holandês) mantidos em regime de pastoreio permanente em região de baixada, correspondente ao clima Aw, no Estado do Rio de Janeiro. Os animais tinham entre 6 e 9 meses de idade, e o experimento teve duração de 24 meses. Os animais eram portadores de infecção natural por diversas espécies de helmintos e o trabalho baseou-se na contagem de ovos por grama de fezes e necropsias de pelo menos quatro animais a cada 28 dias. Observou-se a tendência dos animais abrigarem maiores populações de helmintos nas estações de outono e primavera. Os parâmetros bioclimatográficos representados por elipse, obtida por meio da relação precipitação pluviométrica e temperatura média das mínimas, foram eficientes para demonstrar o potencial de parasitose clí-

\footnotetext{
${ }^{1}$ Aceito para publicação em 4 de outubro de 2002.

${ }^{2}$ Pesquisador visitante da Universidade Federal Rural do Rio de Janeiro (UFRRJ) e Bolsista da Fundação de Amparo à Pesquisa do Estado do Rio de Janeiro (FAPERJ).

${ }^{3}$ Departamento de Epidemiologia e Saúde Pública, Instituto de Veterinária da UFRRJ, Km 47 da antiga Estrada Rio-São Paulo. Cx. Postal 74.548, Seropédica, RJ 23851-970, Brasil. Bolsista do CNPq. E-mail: adivaldo@ufrrj.br
}

nica, a qual correspondeu às estações de outono e primavera.

TERMOS DE INDEXAÇÃO: Epidemiologia, helmintoses pulmonares e gastrintestinais, Haemonchus placei, Cooperia sp, Oesophagostomum radiatum, bezerros.

Classificação CNPq: 5.05.02.00-0 Medicina Veterinária Preventiva.

\section{INTRODUÇÃO}

Os helmintos pulmonares e gastrintestinais constituem-se em agentes etiológicos de significativa importância para a diminuição da produtividade na pecuária bovina (Reinecke 1989). As variações sazonais na dinâmica das populações dos helmintos são reguladas, principalmente, pelas condições climáticas sobre os estágios de vida livre, pela raça e pela susceptibilidade individual do hospedeiro. A interação hospedeiro versus parasito é influenciada pela precipitação pluvial, faixas climáticas favoráveis, concentração de animais por área, faixa etária e índice nutricional (Gibbs 1973).

Segundo Kate (1965), o potencial biótico das helmintoses de ruminantes, quando estudado e conhecido em uma determinada região, torna possível estabelecer o seu modelo estacional, visando as dosificações estratégicas e táticas de controle. Levine (1963), ao estudar o desenvolvimento e sobrevivência das larvas de Haemonchus contortus nas pasta- 
gens, verificaram que $50 \mathrm{~mm}$ de precipitação e 15 a $37^{\circ} \mathrm{C}$ de temperatura média mensal indicavam ótimas condições para a transmissão deste parasito. No entanto, apenas os meses integrados por uma elipse no bioclimatograma apresentavam melhor período de potencial de transmissão.

Os métodos de controle de helmintoses utilizados no Estado do Rio de Janeiro têm sido baseados no uso de antihelmínticos, com potencial estabelecimento de populações resistentes aos princípios ativos disponíveis no mercado. 0 sistema de manejo zootécnico e sanitário, associado aos estudos epidemiológicos, podem minimizar o uso de produtos químicos.

As regiões de baixadas do Estado do Rio de Janeiro correspondem à extensa área fisiográfica, abrangendo a região Metropolitana, Baía da Ilha Grande, Baixadas Litorâneas e parte das regiões Norte e Nordeste, coincidindo com o tipo climático Aw, segundo a classificação de Köppen. Essa região é caracterizada por inverno seco e verão chuvoso e quente, com a temperatura média das médias do mês mais quente superior a $33^{\circ} \mathrm{C}$, e a precipitação média no verão é de 739 $\mathrm{mm}$, e no inverno, de $128 \mathrm{~mm}$.

O objetivo do presente trabalho foi estudar a epidemiologia das helmintoses de bezerros mestiços, em região correspondente ao clima Aw, no Estado do Rio de Janeiro.

\section{MATERIAL E MÉTODOS}

\section{Local e condições climáticas}

Os trabalhos de campo foram conduzidos em piquetes da Embrapa, Projeto de Saúde Animal - Convênio Embrapa/Universidade Federal Rural do Rio de Janeiro, Km 47 da antiga Rodovia Rio - São Paulo (latitude sul $22^{\circ} 48^{\prime}$ e longitude oeste e $43^{\circ} 41^{\prime}$, e altitude de $33 \mathrm{~m}$ ), situado na microrregião Metropolitana do Estado do Rio de Janeiro.
O solo predominante é do tipo franco argiloso arenoso. O clima na região pertence à Classe Aw, segundo a classificação de Köppen. Os dados sobre as variáveis climáticas do período compreendido entre dezembro de 1977 e novembro de 1979, foram fornecidos pelo posto de meteorologia Ecologia Agrícola do $\mathrm{Km} \mathrm{47,} \mathrm{distante} \mathrm{cerca} \mathrm{de} 1 \mathrm{~km}$ do local do estudo. A temperatura do bolo fecal foi obtida com a introdução do bulbo de termômetro de mercúrio, pelo período de 3 minutos e procedida leitura diretamente na escala em ${ }^{\circ} \mathrm{C}$.

\section{Estudo da sazonalidade para animais a campo}

Foram utilizados um total de 98 bezerros desmamados, machos, mestiços (Zebú x Holandês), de 6 a 9 meses de idade, pertencentes ao rebanho da Embrapa Km 47 (antigo Núcleo de Pesquisa em Saúde Animal) ou da Estação Experimental de Itaguaí, Pesagro-Rio, e portadores de infecção natural por diversas espécies de helmintos. O estudo teve a duração de 24 meses e iniciou-se em dezembro de 1977. Os animais permaneciam pelo menos 60 dias em piquetes de 10 ha, de topografia levemente acidentada, com capim Digitaria decumbens (transvala), antes de serem necropsiados. A lotação adotada foi de uma cabeça por hectare, seguindo a tradição local. A medida que foram necropsiados, foram substituídos por outros, da mesma procedência e idade. Os animais não foram submetidos à rotação de pastagem, não receberam suplementação alimentar e não foram medicados.

Utilizaram-se, para a contagem de ovos por grama de fezes (OPG.), a técnica de Gordon \& Whitlock (1939), e para a obtenção das larvas infectantes de Dictyocaulus viviparus e de Strongyloidea, as técnicas de Ueno (1968) e Roberts \& O'Sullivan (1950), respectivamente, e a chave de Keith (1953) para a identificação das formas infectantes. A cada 14 dias foram sacrificados, no mínimo, dois animais. Os animais escolhidos para a necropsia foram os de maior permanência no campo e de maior OPG.

Após o sacrifício dos animais, cada porção do tubo digestivo foi separada e aberta em bandeja. Para os pulmões, procedeu-se a técnica de perfusão, segundo Eckert \& Inderbitzin (1978) e coletaram-se as

Quadro 1. Prevalência, intensidade média de infecção e amplitude total das infecções por helmintos em bezerros em regime de pasto na região litorânea do Estado do Rio de Janeiro

\begin{tabular}{|c|c|c|c|c|c|}
\hline \multirow[b]{2}{*}{ Estações do ano e parâmetros } & \multicolumn{5}{|c|}{ Nematóides } \\
\hline & Haemonchus & Cooperia & Bunostomum & Oesophagostomum & Trichuris \\
\hline \multicolumn{6}{|l|}{ VERÃO } \\
\hline Prevalência (\%) & 100 & 100 & 18 & 91 & 54 \\
\hline Intensidade média entre bezerros parasitados & 559 & 7.262 & 5 & 103 & 16 \\
\hline Intensidade média entre bezerros necropsiados & 559 & 7.262 & 1 & 95 & 9 \\
\hline Amplitude entre bezerros parasitados & $6-4806$ & $5-111666$ & $0-13$ & $0-613$ & $0-65$ \\
\hline \multicolumn{6}{|l|}{ OUTONO } \\
\hline Prevalência (\%) & 100 & 100 & 8 & 96 & 70 \\
\hline Intensidade média entre bezerros parasitados & 1.189 & 12.541 & 1 & 98 & 20 \\
\hline Intensidade média entre bezerros necropsiados & 1.189 & 12.541 & 0 & 94 & 14 \\
\hline Amplitude entre bezerros parasitados & $6-6721$ & $87-69115$ & $0-1$ & $0-396$ & $0-53$ \\
\hline \multicolumn{6}{|l|}{ INVERNO } \\
\hline Prevalência (\%) & 100 & 100 & - & 80 & 65 \\
\hline Intensidade média entre bezerros parasitados & 752 & 13.124 & - & 169 & 24 \\
\hline Intensidade média entre bezerros necropsiados & 752 & 13.124 & - & 135 & 16 \\
\hline Amplitude entre, bezerros parasitados & $3-2703$ & 23- 12050 & - & $0-802$ & $0-100$ \\
\hline \multicolumn{6}{|l|}{ PRIMAVERA } \\
\hline Prevalência (\%) & 96 & 100 & 14 & 89 & 68 \\
\hline Intensidade média entre bezerros parasitados & 1.720 & 4.517 & 4 & 97 & 30 \\
\hline Intensidade média entre bezerros necropsiados & 1.658 & 4.517 & 1 & 88 & 20 \\
\hline Amplitude entre bezerros parasitados & $0-15712$ & 28- 19605 & $0-5$ & $0-477$ & $0-90$ \\
\hline
\end{tabular}


formas adultas e imaturas de D. viviparus. O conteúdo de cada segmento do tubo digestivo, mais o lavado da mucosa era completado para 2 litros com solução fisiológica ou mais, de acordo com o volume do conteúdo do órgão. A mucosa era raspada e digerida em solução de ácido clorídrico a $1 \%$ e mantida na estufa a $40^{\circ} \mathrm{C}$ pelo período de 2 horas. A seguir, eram retiradas duas alíquotas de $10 \%$ do material procedente do abomaso, do intestino delgado e do material digerido e todos, individualmente, foram fixados em formol, a $5 \%$ a $2^{\circ} \mathrm{C}$. Para o intestino grosso, o procedimento utilizado foi a contagem total dos helmintos presentes. Para contagem dos especimens dos gêneros Haemonchus e Cooperia, utilizou-se a técnica descrita por Reinecke (1972). Para os gêneros Trichostrongylus, Bunostomum, Oesophagostomum, Trichuris e para cestódeos, procedeuse a contagem de todos os helmintos presentes. Todo o material fixado foi passado separadamente em tamis com malhas de $145 \mu \mathrm{m}$ de abertura, para formas adultas, e de $37 \mu \mathrm{m}$, para formas imaturas.

\section{RESULTADOS}

Foram estudadas as populações de D. viviparus, Haemonchus placei, Trichostrongylus axei, Cooperia punctata, Cooperia spatulata, Strongyloides papillosus, Bunostomum phlebotomum, Oesophagostomum radiatum, Trichuris spp e Moniezia bene-deni.

O número total de helmintos adultos encontrados durante o período de estudo foi de 979.777 e os mais prevalentes foram: 862.230 (88\%) Cooperia spp, $103.410(10,6 \%)$ H. placei, 9.655 $(0,9 \%)$ de 0 . radiatum. Outras espécies presentes incluíram o achado esporádico de $D$. viviparus, T. axei, S. papillosus, $B$. phebotomum, Trichuris spp e M. benedeni.

A prevalência, a intensidade média de infecção entre os bezerros necropsiados e parasitados, a amplitude das infecções por nematóides nos bezerros, bem como sua distribuição estacional, estão relacionados no Quadro 1 . Foi constatada a presença de $H$. placei, em todos os meses do ano, porém sua ocorrência em intensidade mais elevada entre bezerros parasitados e entre bezerros necropsiados, atingindo níveis de carga parasitária patogênica, foi verificada nas estações de outtono e primavera. Os parâmetros climatológicos e a distribuição sazonal dos helmintos estão demonstrados na Figura 1.

\section{DISCUSSÃO}

As populações de H. placei, Cooperia spp e $O$. radiatum tiveram as maiores prevalências durante as estações de outono e primavera, na região estudada. Os animais em regime de pastoreio permanente tiveram cargas cumulativas de $\mathrm{H}$. placei, obtidas em março/ abril, atingiram seu ápice em maio, com decréscimo da população no período seco e novo ápice em outubro/dezembro.

A temperatura ideal para desenvolvimento larval da maioria das espécies no microclima em nível da vegetação está entre 22 e $26^{\circ} \mathrm{C}$. Reinecke (1970) observou que chuvas bem distribuídas no outono em regiões com ocorrência de verão chuvoso, houve estímulo para o desenvolvimento de larvas de Haemonchus sp e Oesophagostomum sp.

As formas adultas de Cooperia spp estavam presentes durante todas as estações do ano, com aumento da prevalência no outono e primavera. Este helminto apresenta sua maior prevalência entre as faixas etárias de 4 a 6 meses de idade e requer menor índice pluviométrico que outros helmintos gastrintestinais. Larvas de Cooperia spp são mais adaptadas aos extremos de temperatura e dessecação, e todos os meses apre- sentam temperaturas favoráveis ao desenvolvimento das fases infectantes deste parasito no Estado do Rio de Janeiro (Braga 1986, Pimentel Neto et al. 2000).

Ao examinar a distribuição estacional dos helmintos gastrintestinais na região de clima Aw no Estado do Rio de Janeiro, observa-se que durante o verão o número de helmintos foi pequeno e decorrente, provavelmente, das temperaturas elevadas sobre as larvas de pasto e do bom estado
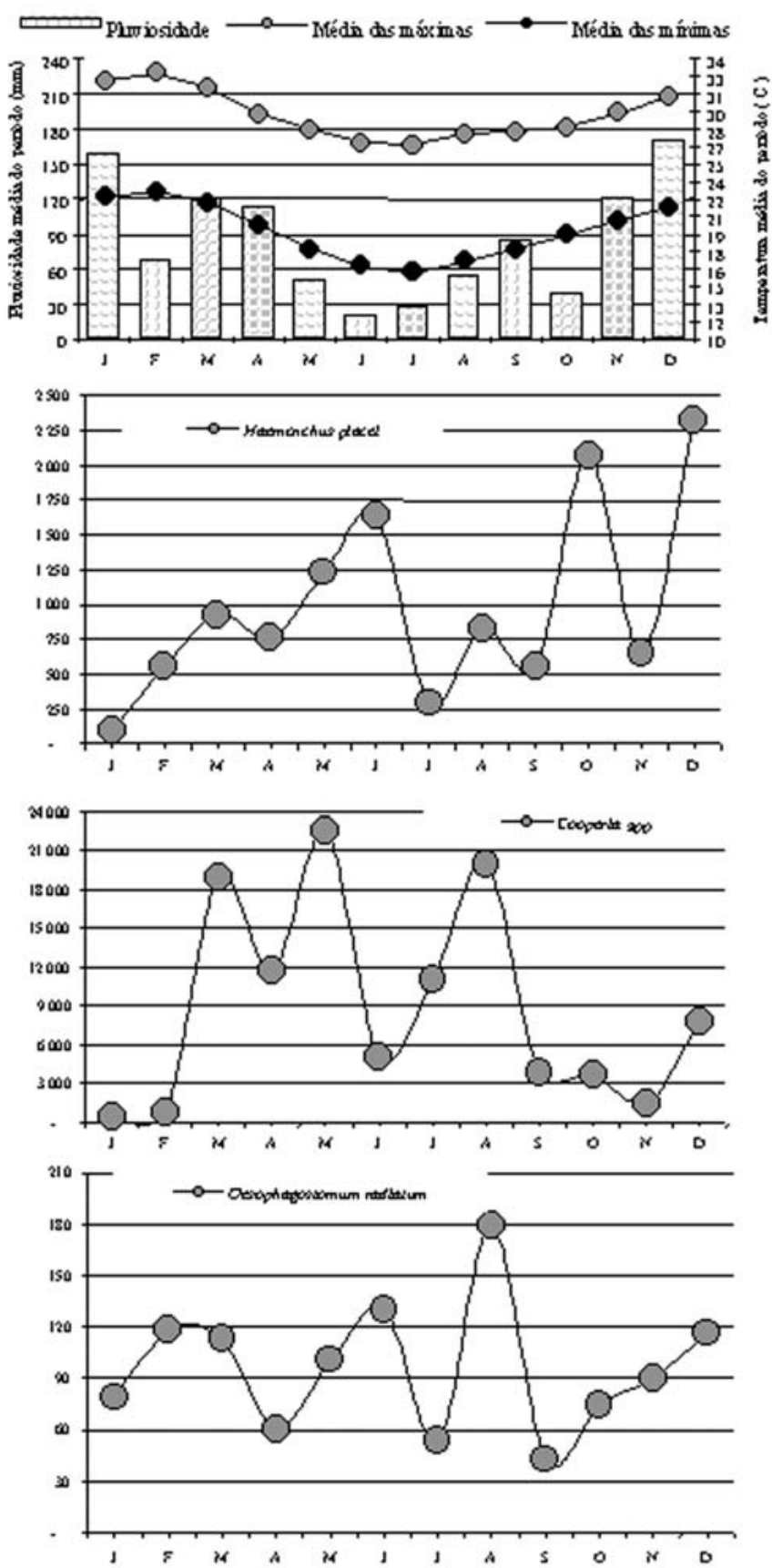

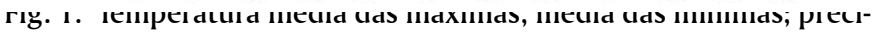
pitação mensal equivalentes ao período do experimento, registradas no posto de meteorologia - Ecologia Agrícola, Seropédica, RJ. Distribuição sazonal das formas adultas de diferentes helmintos parasitos de bezerros na região litorânea do Estado do Rio de Janeiro. 
Quadro 2. Capacidade de sobrevivência de helmintos com potencial para diminuição da produtividade em bezerros no tipo climático Aw, no Estado do Rio de Janeiro (Classificação de Köpen)

\begin{tabular}{lll}
\hline \multirow{2}{*}{ Tipo de clima Aw } & \multicolumn{2}{c}{ Sobrevivência no ambiente } \\
\cline { 2 - 3 } & \multicolumn{1}{c}{ Ótima $^{1}$} & Mínima ou nenhuma \\
\hline & & Dictyocaulus viviparus \\
Clima tropical, inverno & Haemonchus placei & Trichostrongylys axei \\
seco com menos de & Cooperia sp & Bunostomum plebotomum \\
60 mm precipitação e & Oesophagostomum radiatum & Moniesia benedeni \\
verão chuvoso & & Trichuris sp
\end{tabular}

${ }_{1}^{1}$ Helminto recuperado pelo período de dois ou mais meses seguidos.

$\mathbf{2}_{\text {Helminto recuperado esporadicamente. }}$

nutricional dos animais nessa época do ano. Segundo Smeal et al. (1980), as chuvas causam desintegração do bolo fecal, e os estágios infectantes são submetidos aos efeitos deletérios do calor e da luz solar. Braga (1986) e Pimentel Neto et al. (2000) constataram que a permanência de larvas infectantes de T. axei, Haemonchus spp, $O$. radiatum e Cooperia spp nas pastagens foram significativamente menores nos meses de janeiro e fevereiro. Durie (1961) constatou também a diminuição de larvas no pasto no meio das estações do verão e do inverno. No Quadro 2 está representada a capacidade de sobrevivência das formas infectantes dos diferentes helmintos nas regiões de baixadas do Estado do Rio de Janeiro.

Durante o verão, as médias mensais das temperaturas máximas foram de $33,1^{\circ} \mathrm{C}$, e as temperaturas das fezes, observadas diretamente, variaram de $36^{\circ} \mathrm{C}$ a $40^{\circ} \mathrm{C}$. As máximas absolutas durante o verão podem atingir $42^{\circ} \mathrm{C}$ nas regiões litorâneas do Estado do Rio de Janeiro. As elevadas temperaturas alcançadas nas fezes durante o verão, na região estudada, constituem-se em fator limite para o desenvolvimento e sobrevivência das larvas no pasto. É provável que, na ocorrência de precipitações regularmente distribuídas durante o verão, diminuindo a média das máximas, possam haver condições favoráveis para o estabelecimento de parasitose clínica. Salienta-se ainda, que, durante as duas estações de verão, $75 \%$ do rebanho bovino apresentou bom estado nutricional.

Guimarães (1972) promoveu contaminação das pastagens com fezes de bovinos e recuperou maior número de larvas infectantes no mês de novembro. Nos meses de dezembro e janeiro, apesar da temperatura e precipitação pluviométrica estarem acima das exigências mínimas para o desenvolvimento dos estágios pré-infectantes, não houve recuperação das mesmas. Estas também não foram recuperadas durante o período de maio a outubro.

No presente trabalho foi observada prevalência acima de $83,33 \%$ de 0 . radiatum em todas estações do ano, com a média de 623,2 parasitos por animal, o que demostra a importância desse helminto para bezerros na região estudada. 0 . radiatum é um nematóide que interfere na produtividade do rebanho no que se refere à conversão e ganho de peso (Bulman et al. 2001).

O parasitismo por T. axei, C. spatulata, Bunostomum spp, $M$. benedeni e Trichuris spp foi discreto, com ligeira prevalência nos períodos de outono e inverno.

$O$ estabelecimento de parasitose em bezerros guarda relação com a idade do animal, com o gênero de helminto, e com a classe climática local. Guimarães et al. (1975) constataram que bezerros com idade de 3 a 4 meses estavam parasitados predominantemente por $S$. papillosus e que o parasitismo por Haemonchus sp e Trichostrongylus sp apresentou tendência de aumento na faixa etária de 10 a 12 meses; além disso Cooperia spp e Oesophagostomum sp foram encontrados em bezerros com até 16 meses, com brusca redução da intensidade da carga parasitária a partir desta faixa etária.

A temperatura média das máximas e média das mínimas, observadas durante as estações de verão, outono, inverno e primavera, foram respectivamente: $33,2^{\circ} \mathrm{C}$ e $22,1^{\circ} \mathrm{C} ; 27,8^{\circ} \mathrm{C}$ e $17,3^{\circ} \mathrm{C} ; 28,4^{\circ} \mathrm{C}$ e $16 ; 29,9^{\circ} \mathrm{C}$ e $19,3^{\circ} \mathrm{C}$. A precipitação média para as estações de verão, outono, inverno e primavera foram respectivamente: $130,1 \mathrm{~mm} ; 53,7 \mathrm{~mm} ; 31,1 \mathrm{~mm} ; 137,6 \mathrm{~mm}$.

A maior prevalência e intensidade média de parasitismo pelas formas adultas, imaturas e ovos nas fezes foram observadas no período do outono e primavera. A representação gráfica da elipse, obtida pela relação precipitação pluviométrica e temperatura média das mínimas, foi eficiente para demonstrar o potencial de parasitose clínica (Fig. 2). A interação dos parâmetros temperatura ambiente, índice pluviométrico e más condições físicas dos animais foi eficiente para desencadear surtos de parasitose clínica na região. Ocorreram morte por elevadas cargas parasitárias de três animais nos meses de junho e três no mês de agosto de 1978.

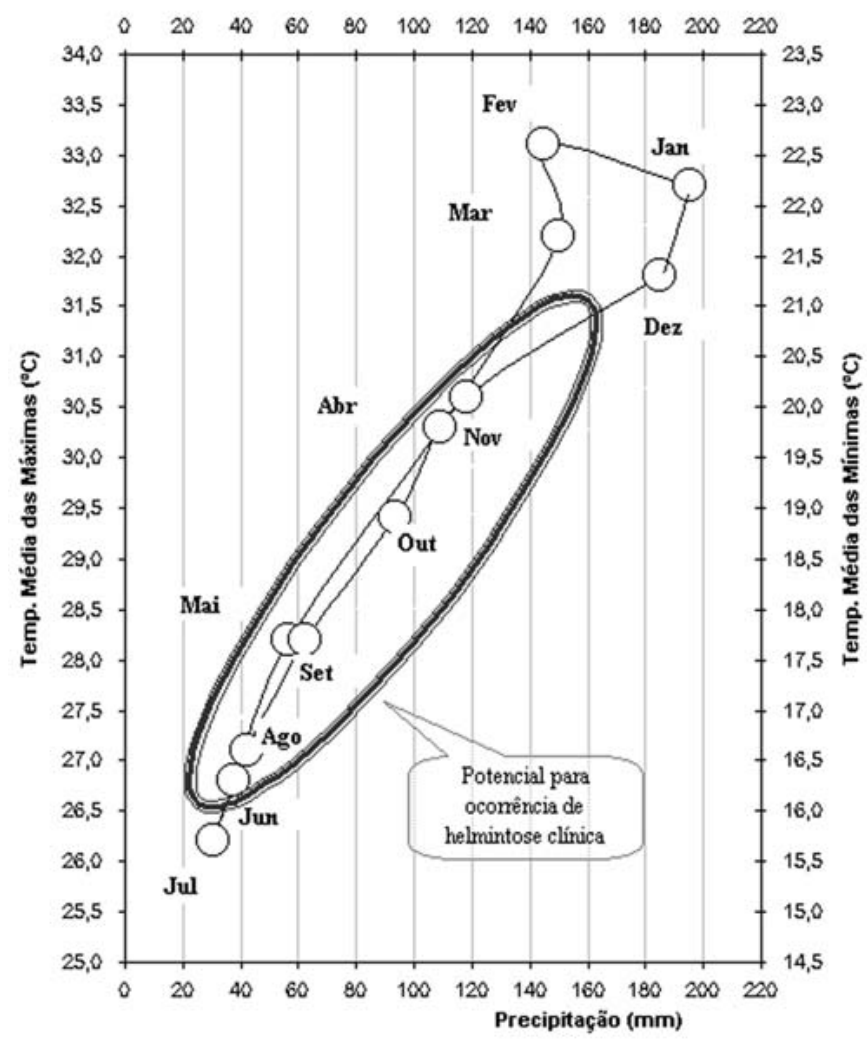

Fig. 2. Mapa bioclimatográfico representativo das condições favoráveis para corrência de parasitose clínica na região Litorânea, no Estado do Rio de Janeiro. Eixo das ordenadas corresponde à temperaturas médias das mínimas; e das máximas e eixo das coordenadas, corresponde à precipitação mensal média entre os anos de 1961 e 1990 registradas no posto de meteorologia Ecologia Agrícola, Seropédica RJ. 


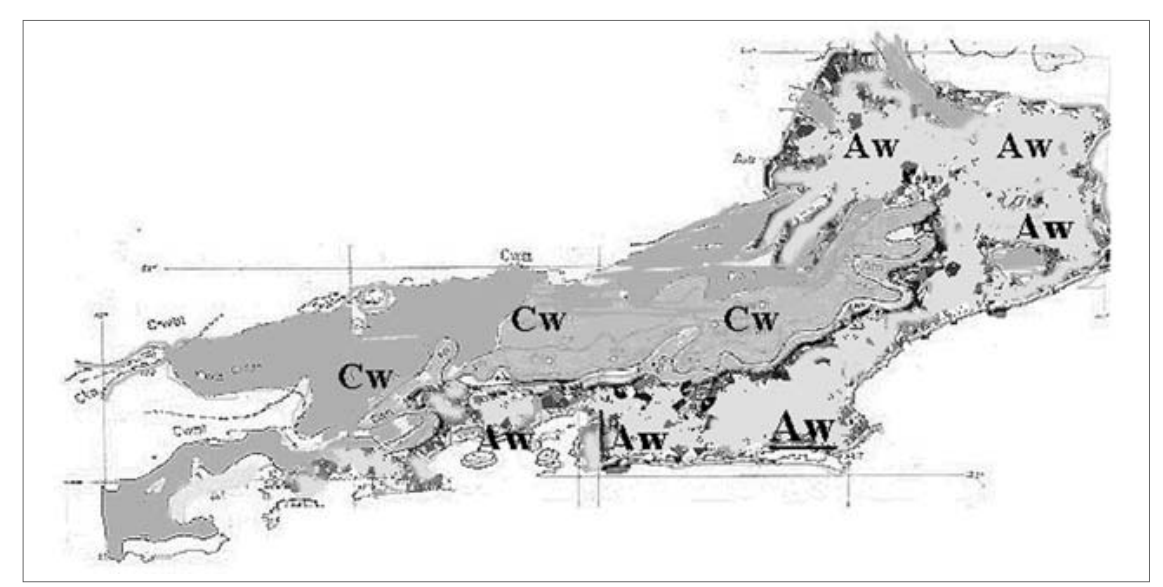

Fig. 3. Mapa climatológico do Estado do Rio de Janeiro, segundo Köpen. O padrão climático tipo Aw, inclui as regiões: Metropolitana, Baía da Ilha Grande, Baixadas Litorâneas e parte das regiões Norte e Nordeste.

No presente trabalho foi possível observar que, na elaboração dos mapas bioclimatográficos, o uso das médias mensais das temperaturas mínimas mostrou ser um modelo adequado para a evidenciação do quadro epidemiológico, visando ao prognóstico das helmintoses de bovinos na região (Fig. 2). Segundo Swan (1970), quando a média mensal das máximas excede $17,2^{\circ} \mathrm{C}$ e $18,3^{\circ} \mathrm{C}$ e ocorre larga amplitude de temperatura, recomenda-se o uso das médias mensais das mínimas. Já Pimentel Neto (1976) utilizou o modelo de elipse para estabelecer o prognóstico da ocorrência de surtos de $H$. placei em região de clima Cwa no estado do Rio de Janeiro. Pesquisas conduzidas em regiões de clima Cw por Oliveira (1988), Furlong et al. (1985) e Pimentel Neto \& Fonseca (1999) indicam que nessa região a estação de inverno corresponde ao período crítico para infecção por helmintoses gastrintestinais, particularmente por $\mathrm{H}$. placei.

Segundo Gordon (1963) e Pimentel Neto (1976), o bioclimatograma permite perceber o potencial para ocorrência de parasitose clínica em determinada região, a época do ano e indicar, ao mesmo tempo, quais parasitos podem ocorrer em clima similar. $O$ verão com altas temperaturas, $\mathrm{o}$ inverno seco e o solo predominantemente arenoso das regiões de baixada no Estado do Rio de Janeiro (Fig. 3) contribuem para que haja rápida evaporação e conseqüiente diminuição do potencial de ocorrência de parasitose clínica no meio dos períodos de verão e de inverno.

Agradecimentos.- Os autores manifestam seus agradecimentos ao Dr. Silvio Cardoso Romero, pela efetiva colaboração na elaboração deste trabalho.

\section{REFERÊNCIAS}

Braga R.M. 1986. Sobrevivência de larvas infectantes de nematódeos gastrintestinais de bovinos, sob condições naturais. Revta Bras. Med. Vet. 8:186-188.

Bulman M.A., Pimentel Neto M.\& Fonseca A.H. 2001. Oesofagostomose experimental em bezerros. Revta Bras. Ciênc. Vet. 8:47-50.

Durie P.H. 1961. Parasitic gastro-intestinal of cattle the distribution and survival of infective strongyle larvae on pasture. Aust. J. Agric. Res. 12:1200-1211.

Eckert J. \& Inderbitzin F. 1978. Arrested development of Dictyocaulus viviparus in cattle and the effect of febendazole against inibited stages. In: III Workshop on "Arrested Development of Nematodes in Sheep and Cattle". Facts and reflections. Lelysted, Netherlands, p. 125-135.

Furlong J., Abreu H.G.L. \& Verneque R.S. 1985. Parasitoses dos bovinos na região da Mata de Minas Gerais. 1. Comportamento estacional de nematódeos gastrointestinais. Pesq. Agropec. Bras. 20(1):143-153.

Gibbs H.C. 1973. Transmission of parasites with reference to the strongyles of domestic sheep and cattle. Can. J. Zool. 51:281-289.

Gordon H. McL. \& Whitlock H.V. 1939. A new technique for couting nematode eggs in sheep faeces. J. Counc. Sci. Ind. Res. Aust. 12:50-52

Gordon H. McL.1963. Helminthic diseases of sheep in Australia. Vet. Med. Nachr. 2/3:9-30.

Guimarães M.P. 1972. Variação estacional de larvas infectantes de nematóides parasitos de bovinos em pastagem de cerrado de Sete Lagoas, MG. Arqs Esc. Vet. UFMG 24(1):97-113.

Guimarães M.P., Freitas M.G., Costa H.M.A. \& Costa J.O. 1975. Intensidade parasitária por nematóides no tubo digestivo de bezerros em diferentes faixas etárias. Arqs Esc. Vet. UFMG 27(1):67-72.

Kate R.C. 1965. Ecological aspects of helminth transmission in domesticated animals. Am. Zoologist 5:95-130.

Keith R.K. 1953. The differentiation of the infective larvae of some common nematode. Aust. J. Zool. 1:223-235.

Levine N.D. 1963. Weather, climate and the bionomics of ruminant nematode larvae. Adv. Vet. Sci. 8:215-261.

Pimentel Neto M. 1976. Epizootiologia da haemoncose em bezerros de gado de leite no estado do Rio de Janeiro. Pesq. Vet. Bras., Sér. Vet. 11:101-114.

Pimentel Neto M. \& Fonseca. A.H. 1999. Epidemiologia das helmintoses de helmintos pulmonares e gastrintestinais de ruminantes de bovinos de leite, na microrregião homogênea do Vale do Paraíba Fluminense. Hora Vet., Porto Alegre, 112:41-46.

Pimentel Neto M., Ribeiro, M.C. \& Fonseca A.H. 2000. Distribuição sazonal e longevidade das larvas infestantes de nematódeos gastrintestinais de bovinos em pastagens na Baixada Fluminense, Rio de Janeiro. Revta Bras. Ciênc. Vet. 7:37-41.

Oliveira G.P. 1988. Epidemiologia dos nematódeos gastrintestinais de bovinos leiteiros no município de São Carlos, estado de São Paulo. Pesq. Agropec. Bras. 23(2):189-195.

Reinecke R.K. 1970. Helminth diseases in domestic animals in relation to their environments. S. Afr. J. Sci. 66:192-198.

Reinecke R.K.1972. An anthelmintic test for gastro-intestinal nematodes of cattle. Onderstepoort. J. Vet. Res. 39:153-178.

Reinecke R.K.1989. Veterinary Helmintology. Butterworths, Durban. 392p.

Roberts F.H.S. \& O'Sullivan P.J. 1950. Methods for eggs counts and larval cultures for strongyles infecting the gastro-intestinal tract of cattle. Aust. J. Agric. Res. 1:99-192.

Smeal M.G., Robinson G.G. \& Freaser G.C. 1980. Seasonal availability of nematode larvae on pasture grazed by cattle in New South Wales. Aust. Vet. J. 56:74-79.

Swan R.A.1970. The epizootiology of haemonchosis in sheep. Aust. Vet. J. 46:485-492.

Ueno H.1968. A simple method for detection of Dictyocaulus viviparus larvae in faeces of cattle 1. Procedure and basic tests. Jap.Vet. Assoc. 21:210-213. 